\title{
Epidemiological and Clinical Features of the Preterms Followed-Up at Our Clinic in Turkey: A Study about 458 Infants
}

\author{
Muberra Akdogan', Mehmet Cem Sabaner'1, Veysel Levent Karabas² \\ ${ }^{1}$ Department of Ophthalmology, Afyon Kocatepe University, Afyon, Turkey \\ ${ }^{2}$ Department of Ophthalmology, Kocaeli University, Kocaeli, Turkey \\ Email: mbrakdogan@yahoo.com
}

How to cite this paper: Akdogan, M., Sabaner, M.C. and Karabas, V.L. (2018) Epidemiological and Clinical Features of the Preterms Followed-Up at Our Clinic in Turkey: A Study about 458 Infants. Open Journal of Ophthalmology, 8, 214-223. https://doi.org/10.4236/ojoph.2018.84025

Received: October 8, 2018

Accepted: November 6, 2018

Published: November 9, 2018

Copyright (c) 2018 by authors and Scientific Research Publishing Inc. This work is licensed under the Creative Commons Attribution International License (CC BY 4.0).

http://creativecommons.org/licenses/by/4.0/

\begin{abstract}
Purpose: We aimed to evaluate 458 infants, who were followed up due to retinopathy of prematurity (ROP), demographically and epidemiologically. Methods: 458 infants with ROP, who were referred from our hospital's neonatal intensive care unit and from other health centers to our clinic for the staging and treatment between February 2016 and December 2017, were studied retrospectively. Design: Retrospective-observational study. Results: Of the 458 infants, 243 infants $(53.06 \%)$ did not have ROP and they were at a mean of 32nd (26 - 35) gestational week, weighing a mean of 1700 (820 $2830)$ gr. The remaining 215 (46.94\%) infants were diagnosed with ROP at various stages and they were at a mean of 29th (23 - 35) gestational week, weighing a mean of $1180(490$ - 2850) gr. Of the infants without ROP, 116 $(47.7 \%)$ were females and $127(52.3 \%)$ were males. The mean duration of stay at the incubator was a mean of $16.5(0-120)$ days and the mean duration of follow-up was a mean of 43 (40 - 56) weeks. $103(40.2 \%)$ of them were breastfed only. Of the infants with ROP at various stages, 95 (44.2\%) were females, and 120 (55.8\%) were males. The mean duration of stay at the incubator was 42 (6 - 195) days, and the mean duration of follow-up was 48 (40 73) weeks. $43(20.0 \%)$ of them were breastfed only. 32 (13.2\%) infants without ROP were born of multi-fetal pregnancies. Only 1 of them was born as one of the triplets. Of the babies with ROP; 56 (26.04\%) were born of multi-fetal pregnancies, 5 of them being triplets. The numerical values of the gestational week at birth and the birth weight, and the rates of breastfeeding were lower in infants with ROP compared to the ones without it ( $\mathrm{p}<0.001)$. However, their follow-up durations were longer $(p<0.001)$. There was observed a male predominance and multi-fetal pregnancies in the infants with ROP (respectively, $\mathrm{p}>0.05,<0.001)$. Conclusions: This study showed that the gestational
\end{abstract}


age, birth weight, breastfeeding rates, birth history and follow-up periods of multiple fetal pregnancies were different in ROP infants compared to non-ROP infants.

\section{Keywords}

Retinopathy of Pematurity, Preterm, Intravitreal Injection, Laser

Photocoagulation

\section{Introduction}

Retinopathy of prematurity (ROP), is the major cause of visual impairment and blindness in neonates worldwide. A demographic census in 2010 reported 184,700 preterm infants worldwide with ROP; 20,000 of them were blind or serious visually impaired [1]. This problem is reaching epidemic ratios in middle-income and developing countries; the survival of extremely premature babies is increasing without a significant change in morbidity [2]. The ROP was first recognized as an important cause of blindness in high-income countries in the 1940s and 1950s when survival ratios of very-low-birth-weight babies $<1500 \mathrm{~g}$ or approximately 32-weeks gestation age (GA) were improving in association with the widespread use of unrestricted oxygen support. This has been termed as the first ROP epidemic, which affected preterm infants in high-income countries [1]. The country-based studies of ROP have been showed in different countries, with different outcomes reported [3] [4] [5] [6] [7]. In the USA, Lad et al. showed that the incidence of ROP was $15.58 \%$ in premature babies from 1997 to 2005 [3] [6]. In the UK, the yearly ROP incidence was $1.28 \%$ in 1990 and $12.55 \%$ in 2011 in infants born weighing less than $1500 \mathrm{~g}$ [5]. In the Netherlands, the reported incidence of ROP in infants with GA less than 32 weeks or birth weight (BW) less than $1500 \mathrm{~g}$ was $21.9 \%$ [4]. In South India, reported that the incidence of ROP was $32.6 \%$ and was strongly associated with low BW [8]. In Sweden, a high incidence of ROP of up to $72.7 \%$ showed in premature infants with GA less than 27 weeks [7]. In China, reported the incidence of ROP development was $60.7 \%$ and a lighter BW and lower GA were the only common independent risk factors for both ROP and Type 1 ROP [9]. The most commonly reported risk factors were low BW and GA [3] [10]-[15].

\section{Material and Methods}

458 infants with ROP, who were referred from our hospital's neonatal intensive care unit and from other health centers to our clinic for stages and treatment between February 2016 and December 2017, were studied retrospectively. HSU Bursa Yuksek Ihtisas Training and Research Hospital Ethics Committee was approved this study, and principles of the Declaration of Helsinki were followed. Within the scope of the study, the GA is between 23 - 35 weeks and babies whose $\mathrm{BW}$ is 2850 grams or fewer records were reviewed retrospectively. 
Infants' pupils were dilated with instillation $0.5 \%$ tropicamide and $1 \%$ phenylephrine topical eye drops for 3 times for 5 minutes before examination. After proper pupillary dilatation, topical anesthesia was applied with proparacaine hydrochloride (Alcaine $e^{\oplus}$, Alcon, USA) done. Once the lids were opened the eye speculum and then, with an indirect ophthalmoscope and +28 dioptric lens, scleral all retinal areas were examined with the aid of a scleral indenter and fundus images were recorded into the Archimedes VGA imaging system (Pronova, Ankara, Turkey). All examinations and treatments were performed by a single physician (MA). Patients without retinopathy were followed every 2 weeks until vascularization is completely complete (approximately until the 45th gestational week). When retinopathy is detected based on the International PR Classification criteria (ICROP), and severity of retinopathy between avascular areas (if demarcation line is present Stage 1, elevation or "ridge" is present Stage 2, if extraretinal fibrovascular proliferation is present Stage 3, If partial retinal detachment involving extrafoveal/fovea is present Stage $4 \mathrm{a} / \mathrm{b}$, if total retinal detachment is present Stage 5), settlement (zone), amount of involvement (clocks) and peripapillary vasculature examination findings by indicating the presence of anomaly (plus) it was noted. In the case of the present findings of these patients according to the plan; infants with ROP were classified into 4 groups according to ICROP classification with regard to the prevalence and severity of retinal proliferation as stage 0 - 1 ROP Group 1, stage 1 - 2 ROP Group 2, stage 2 - 3 and above ROP Group 3, and aggressive posterior ROP (APROP, Type II or rush-type ROP which has the following characteristic: more posterior location; rapid progression rather than the classic stages 1 - 5; and poor prognosis despite early treatment) Group 4 [16] [17] [18] [19]. Only one infant in Group 3 had stage $4 \mathrm{~A}$ ROP and there were no other patients with higher stages of ROP.

We treated infants according to the criteria of the Early Treatment in Retinopathy of Prematurity Study (ETROP) [20].

Fluorescein angiography (FA) was performed using the Retcam3 (Clarity Medical Systems, Pleasanton, CA, USA). FA was performed on patients whose parents approved the FA procedure and carried out in an operating room under intravenous sedation. FA examination was performed with using a bolus of $10 \%$ Fluorescein solution (Fluorescite, Alcon Pharma GmbH, Freiburg, Germany) intravenously administered with a dose of $0.1 \mathrm{~mL} / \mathrm{kg}$ body weight and photographs were recorded in the early, middle, and late phases.

The IVB (intravitreal bevacizumab) dose administered was $0.16 \mathrm{mg}$ (very low dose) for all infants who treated intravitreal therapy. It was administered to all infants under local anesthesia using $0.5 \%$ proparacaine hydrochloride drops (Alcaine $^{\oplus}$, Alcon, USA) in the sterile operating room via $1 \mathrm{~mm}$ posterior to the limbus using a 30-gauge needle. The LPC (laser photocoagulation) was applied with laser device (Iridex; Oculight SL, Mountainview, CA, U.S.A.) in sterile operating room, under sedation or general anesthesia, to the patients who were required on group 2 and above for avascular zones. 
SPSS version 21.0 software for Windows (Statistical Package for the Social Sciences, IBM, Chicago, IL, USA) was used for the data analyses. The MannWhitney $U$ test was used to compare variables. Descriptive statistics are expressed as the frequency and percentage for qualitative data and as the mean \pm standard deviation or median (range) for quantitative data with and without normal distribution, respectively. A P-value $<0.05$ was considered statistically significant.

\section{Results}

Four-hundred and fifty-eight infants with an average GA of 30.98 (range, 23 35) weeks and average BW of 1580 (range, 490 - 2850) grams were enrolled in the study. Various stages of ROP were observed in 215 (46.94\%) infants while no ROP was observed in 243 (53.06\%). Among infants without ROP, 116 (47.7\%) were girls, and 127 (52.3\%) were boys; in those with ROP, 95 (44.2\%) were girls, and 120 (55.8\%) were boys.

Infants who without ROP development were followed for an average of 43 (range, 40 - 56) weeks; those who with ROP develop were followed for an average of 48 (range, 42 - 73) weeks. Infants with and without ROP were kept in an incubator for an average of 42 (range, 6 - 195) and 16.5 (range, 3 - 120) days, respectively. In between two groups; GA, BW, incubation duration time and follow up time differences were statistically significant (all of them; $\mathrm{p}<0.001$ ) (Table 1).

$103(40.2 \%)$ of them were breastfed only. Of the infants with ROP at various stages, $43(20 \%)$ of them were breastfed only. 32 (13.2\%) infants without ROP were born of multi-fetal pregnancies. Only one of them was born as one of the triplets. Of the infants with ROP; 56 (26.04\%) were born of multi-fetal pregnancies, five of them being triplets.

In ROP (+) group; number of patients were in group 1 was 78 (35.6\%), 69 $(32.2 \%)$ in group 2, 29 (13.6\%) in group 3, 39 (18.8\%) in group 4. All of the infants in group 1 showed a regression in ROP stage without treatment. 23 infants

Table 1. Clinical characteristics of the control and study group infants.

\begin{tabular}{cccc}
\hline & Control Group & Study Group & $\mathrm{p}$ \\
\hline Gestational age (week) & $32(26-35)$ & $29(23-35)$ & $<0.001^{*}$ \\
Birth weight (gram) & $1700(820-2830)$ & $1180(490-2850)$ & $<0.001^{*}$ \\
Length of stay in incubator (day) & $16.5(2-120)$ & $1180(490-2850)$ & $<0.001^{*}$ \\
Gender(male/female) & $127 / 116$ & $120 / 95$ & $>\mathbf{0 . 0 5 ^ { + }}$ \\
Following time(day) & $43(40-56)$ & $48(40-73)$ & $<0.001^{*}$ \\
Breastfeding & $103(40.2 \%)$ & $43(20 \%)$ & $<0.001^{+}$ \\
Multi-fetal pregnancies & $32(13.2 \%)$ & $56(26.04 \%)$ & $<0.001^{+}$ \\
\hline
\end{tabular}

*: Mann Whitney-U test, ${ }^{+}$: Chi-square test, and result $\mathrm{p}<0.05$ was considered to be statistically significantly different and was indicated in bold. 
in group $2(33.3 \%)$ were administered IVB, and five of these $(7.2 \%)$ infants were underwent new diode LPC for avascular zones. In group 3, 23 infants (79.3\%) were administered IVB, among whom ten (34.4\%) of these infants were underwent additional LPC for avascular zones. In Group 4, all infants (100\%) were administered IVB, among whom $19(48.7 \%)$ of these infants were underwent additional LPC. In group 4, one eye of an infant with APROP (+) was referred to our clinic from another health center. Vitreoretinal surgery (PPV) was performed at another center for repair the macular hole, but it was not successful surgery. While the other eye of the same infant was showed regression in ROP stage and activity with IVB and LPC therapy. FFA was performed to five patients, and three of them a vascular arrest was seen, the other two patients evaluated as "leakage". Complications such as cataract or endophthalmitis were not observed after LPC or IVB treatment.

\section{Discussion}

ROP affects a substantial number of premature infants worldwide. The negative effects of ROP on vision can be predicted in the premature infant. Early detection and treatment of ROP are crucial to prevent poor vision in these infants so that infants with ROP will have a better quality of life [21]. Even so, the ROP with severe and advantage stages may result in blindness or poor sight even with treatment.

GA, BW, and oxygen therapy are well-known major risk factors in the development of ROP [22] [23] [24]. In this study, the mean of the gestational week at birth, birth weight, and length of stay in incubator were lower in infants with ROP compared to the ones without it, significantly. Additionally, a count of multi-fetal pregnancies was higher in infants with ROP compared to the ones without it, significantly. We foresee that this is due to the birth of a lower birth weight baby due to multiple pregnancies. In additionally, the rates of breastfeeding were lower in infants with ROP compared to the ones without it, significantly. This difference was showed but no conclusions can be drawn on this study on whether that breastfeeding is protective for ROP. Further studies on the effects of breastfeeding on ROP are needed.

In East Asia, Eastern Bloc and Latin American countries, the incidence of ROP development has increased over the years [25] [26] [27] [28] [29]. In the presence of technological and medical developments, decreased mortality but increased morbidity. It is thought that this condition is called in "third epidemic" in developing countries as a result of the increase in premature birth rate due to the widespread use of reproductive techniques and the increase of premature birth rate and the uncontrolled oxygen treatment of infants [1] [23] [27].

For the infants who should be screened in our country, there is not yet a common opinion. Mutlu et al. showed that infants with a gestational age of 32 weeks or less may have a greater risk of developing ROP and screening should be intensified in the presence of risk factors. In addition, Sarikabadayı et al. showed that infants who may be suitable for screening have less than 32 weeks of birth 
and 1500 grams of birth weight. Ugurbaş et al. suggest that the criteria in the United States and Britain have could be adapted to Turkey, and so decrease that the number of unnecessary fundus examinations [30]. Bas et al., in the multicentric study, showed that screening of infants with a GA $\leq 34$ weeks or a BW < $1700 \mathrm{~g}$ appears to be appropriate in Turkey [24]. In our study, there were an average GA of 29 (range, 23 - 35) weeks and average BW of 1180 (range, 490 2850) grams in infants with ROP. Akçakaya et al., below 34 weeks and 2000 grams; Akman et al., below 34 weeks and 1850 grams reported that it would be appropriate to screen the born babies [31] [32]. Sahin et al., extremely premature infants (who was born before 28 weeks) with lower GA were found to be more likely to develop type 1 ROP [33]. In our study, there were 81 (37.6\%) extremely premature infants (28 w and below) and 57 of them (70.4\%) had Type 1 ROP. In additionally, Başmak et al., showed that more mature infants may also develop threshold ROP and require early treatment [34]. Similarly, in our study, infants who required treatment 56 infants (26.2\%) were born at 1500 grams over and 45 infants $(20.9 \%)$ were born at 32 weeks over. The study conducted in high-income countries have shown that babies born at $\geq 32$ weeks are not at high risk for developing ROP and most babies born at $>28$ weeks who develop ROP have a mild disease that spontaneously regressed without any treatment [35]. The TR-ROP study showed comparable to those from other developing countries and showed that more mature and heavier babies were at risk for severe ROP [24] [36]. Heavier and more mature babies can also develop ROP in developing countries because there is insufficient awareness of the risk factors of the disease process, a shortage of skilled professionals and/or a shortage of essential equipment to care for infants [37].

Bas et al. showed that all infants diagnosed with ROP, 16.5\% needed laser photocoagulation, and 20 patients born at $>32$ weeks' GA required this treatment modality. Vitreoretinal surgery was performed in 28 infants with severe ROP: 23 with GA $\leq 28$ weeks and 5 with GA 29 - 32 weeks [38]. In another study of there, all of the infants screened for ROP, $414(6.7 \%)$ needed to treatment [24]. In our work, 85 of the 215 ROP diagnosed infants (39.5\%) needed to be treated with LPC or IVB. Only one eye was needed to perform PPV at another center. As a result, one $(0.22 \%)$ of our 458 babies developed visual loss that could not be prevented, and $82(18.4 \%)$ babies would face serious vision loss if untreated. All of the infants in type 1 (35.6\%) showed a regression without treatment in follow-up in our study. Also, evidence-based data are not available to confirm a favorable risk-benefit ratio of IVB usage in cases earlier than type 1 ROP [39].

The incidence of the ROP in Turkey was reported as $59.0 \%$ in cases with a GA lower than 28 weeks, $24.3 \%$ in cases who were born between 29 and 32 weeks, as well as $6.8 \%$ in cases born between 33 and 36 weeks [40]. These rates were reported as $89.0 \%, 51.7 \%$, and $14.2 \%$, respectively by the ETROP study. Sariaydin et al., reported $78.3 \%, 40 \%$ and $19.4 \%$ in their study [41]. These rates were 
showed as $70.9 \%, 36.7 \%$, and $10.4 \%$, respectively in our study.

The main limitations of the present study were its retrospective design including observation of the data from the only a single center, thereby, interpretation of our findings should be made carefully. However, as our, the ROP specific clinic is one of the biggest referral centers for the ROP treatment in Turkey. In additionally, no current consensus on Turkish ROP screening strategy is present.

In conclusion, this study showed that the lower GA, BW, the rates of breastfeeding, and the higher duration of follow-up, history of birth from multiple fetal pregnancies were different in infants with ROP. Additionally, population-based and multicentral study including epidemiological, demographical information and risk factors for ROP need to be conducted throughout all of Turkey to make screening criteria specific for the Turkish population. A good scanning program will reduce serious vision loss due to ROP. Also, the ophthalmologist and neonatal specialists should work on the correlate, it should be noted that this is a team game.

\section{Conflicts of Interest}

The authors report no conflict of interest. The authors alone are responsible for the content and writing of this article.

\section{Disclosure Statement}

All authors certify that they have no affiliations with or involvement in any organization or entity with any financial interest or non-financial interest in the subject matter or materials discussed in this manuscript.

\section{Ethical Approval}

The study adhered to the tenets of the Declaration of Helsinki. HSU Bursa Yuksek Ihtisas Research and Training Hospital Ethics Committee of Clinical Research approved the study protocol.

\section{Funding}

No funding was received for this research.

\section{References}

[1] Blencowe, H., Lawn, J.E., Vazquez, T., Fielder, A. and Gilbert, C. (2013) Preterm-Associated Visual Impairment and Estimates of Retinopathy of Prematurity at Regional and Global Levels for 2010. Pediatric Research, 74, 35-49. https://doi.org/10.1038/pr.2013.205

[2] Gergely, K. and Gerinec, A. (2010) Retinopathy of Prematurity-Epidemics, Incidence, Prevalence, Blindness. Bratislavske Lekarske Listy, 111, 514-517.

[3] Lad, E.M., Nguyen, T.C., Morton, J.M. and Moshfeghi, D.M. (2008) Retinopathy of Prematurity in the United States. British Journal of Ophthalmology, 92, 320-325. https://doi.org/10.1136/bjo.2007.126201 
[4] Van Sorge, A.J., Termote, J.U.M., Kerkhoff, F.T., Van Rijn, L.J., Simonsz, H.J., Peer, P.G.M., et al. (2014) Nationwide Inventory of Risk Factors for Retinopathy of Prematurity in the Netherlands. The Journal of Pediatrics, 164.

https://doi.org/10.1016/j.jpeds.2013.11.015

[5] Painter, S.L., Wilkinson, A.R., Desai, P., Goldacre, M.J. and Patel, C.K. (2015) Incidence and Treatment of Retinopathy of Prematurity in England between 1990 and 2011: Database Study. British Journal of Ophthalmology, 99, 807-811.

https://doi.org/10.1136/bjophthalmol-2014-305561

[6] Lad, E.M., Hernandez-Boussard, T., Morton, J.M. and Moshfeghi, D.M. (2009) Incidence of Retinopathy of Prematurity in the United States: 1997 through 2005. American Journal of Ophthalmology, 148. https://doi.org/10.1016/j.ajo.2009.04.018

[7] Austeng, D., Källen, K.B.M., Hellström, A., Tornqvist, K. and Holmström, G.E. (2010) Natural History of Retinopathy of Prematurity in Infants Born before 27 Weeks' Gestation in Sweden. JAMA Ophthalmology, 128, 1289-1294.

https://doi.org/10.1001/archophthalmol.2010.234

[8] Nanayakkara, S., Misch, D., Chang, L. and Henry, D. (2013) Depression and Exposure to Suicide Predict Suicide Attempt. Depress Anxiety, 30, 991-996.

https://doi.org/10.1002/da.22143

[9] Yau, G.S.K., Lee, J.W.Y., Tam, V.T.Y., Liu, C.C.L. and Wong, I.Y.H. (2014) Risk Factors for Retinopathy of Prematurity in Extremely Preterm Chinese Infants. In: Turkcu, F.M., Ed., Medicine (United States), 93, e314.

[10] Chiang, M.F., Arons, R.R., Flynn, J.T. and Starren, J.B. (2004) Incidence of Retinopathy of Prematurity from 1996 to 2000: Analysis of a Comprehensive New York State Patient Database. Ophthalmology, 111, 1317-1325.

https://doi.org/10.1016/j.ophtha.2003.10.030

[11] Li, M.L., Hsu, S.M., Chang, Y.S., Shih, M.H., Lin, Y.C., Lin, C.H., et al. (2013) Retinopathy of Prematurity in Southern Taiwan: A 10-Year Tertiary Medical Center Study. Journal of the Formosan Medical Association, 112, 445-453. https://doi.org/10.1016/j.jfma.2012.03.002

[12] Gebeşçe, A., Uslu, H., Keleş, E., Yildirim, A., Gürler, B., Yazgan, H., et al. (2016) Retinopathy of Prematurity: Incidence, Risk Factors, and Evaluation of Screening Criteria. Turkish Journal of Medical Sciences, 46, 315-320. https://doi.org/10.3906/sag-1407-127

[13] Huang, H.C., Yang, H.I., Chou, H.C., Chen, C.Y., Hsieh, W.S., Tsou, K.I., et al. (2015) Preeclampsia and Retinopathy of Prematurity in Very-Low-Birth-Weight Infants: A Population-Based Study. PLoS One, 10. https://doi.org/10.1371/journal.pone.0143248

[14] Su, Y.Y., Wang, S.H., Chou, H.C., Chen, C.Y., Hsieh, W.S., Tsao, P.N., et al. (2016) Morbidity and Mortality of Very Low Birth Weight Infants in Taiwan-Changes in 15 Years: A Population Based Study. Journal of the Formosan Medical Association, 115, 1039-1045. https://doi.org/10.1016/j.jfma.2016.10.011

[15] Hwang, J.H., Lee, E.H. and Kim, E.A.-R. (2015) Retinopathy of Prematurity among Very-Low-Birth-Weight Infants in Korea: Incidence, Treatment, and Risk Factors. Journal of Korean Medical Science, 30, S88. https://doi.org/10.3346/jkms.2015.30.S1.S88

[16] Drenser, K.A., Trese, M.T. and Capone, A. (2010) Aggressive Posterior Retinopathy of Prematurity. Retina, 30, S37-S40. https://doi.org/10.1097/IAE.0b013e3181cb6151

[17] Wilkinson, A.R., Haines, L., Head, K. and Fielder, A.R. (2008) UK Retinopathy of 
Prematurity Guideline. Eye, 23, 2137-2139.

https://doi.org/10.1016/j.earlhumdev.2007.12.004

[18] Shah, P.K. (2016) Retinopathy of Prematurity: Past, Present and Future. World Journal of Clinical Pediatrics, 5, 35-46. https://doi.org/10.5409/wjcp.v5.i1.35

[19] International Committee for the Classification of Retinopathy of Prematurity (2005) The International Classification of Retinopathy of Prematurity Revisited. Archives of Ophthalmology, 123, 991-999. https://doi.org/10.1001/archopht.123.7.991

[20] Good, W.V. (2004) Final Results of the Early Treatment for Retinopathy of Prematurity (ETROP) Randomized Trial. Transactions of the American Ophthalmological Society, 102, 233-248.

[21] Van Sorge, A.J., Schalij-Delfos, N.E., Kerkhoff, F.T., Van Rijn, L.J., Van Hillegersberg, J.L.A.M., Van Liempt, I.L.A., et al. (2013) Reduction in Screening for Retinopathy of Prematurity through Risk Factor Adjusted İnclusion Criteria. British Journal of Ophthalmology, 97, 1143-1147. https://doi.org/10.1136/bjophthalmol-2013-303123

[22] Bancalari, E.H. and Walsh, M.C. (2015) Bronchopulmonary Dysplasia in the Neonate. In: Martin, R.J., Fanaroff, A.A. and Walsh, M.C., Eds., Fanaroff Martin's Neonatal-Perinatal Medicine 2-Volume Set, 1157-1169.

[23] Esen, E., Erdem, E., Yar, K., Demircan, N. and Soylu, M. (2014) Results of Screening for Retinopathy of Prematurity: How the Ideal Screening Program Should Be? Türk Oftalmoloji Dergisi, 44, 42-46. https://doi.org/10.4274/tjo.72621

[24] Bas, A.Y., Demirel, N., Koc, E., Ulubas Isik, D., Hirfanoglu, İ.M. and Tunc, T. (2018) Incidence, Risk Factors and Severity of Retinopathy of Prematurity in Turkey (TR-ROP Study): A Prospective, Multicentre Study in 69 Neonatal İntensive Care Units. British Journal of Ophthalmology.

[25] Hariharan, L., Gilbert, C.E., Quinn, G.E., Barg, F.K., Lomuto, C., Quiroga, A., et al. (2018) Reducing Blindness from Retinopathy of Prematurity (ROP) in Argentina Through Collaboration, Advocacy and Policy Implementation. Health Policy Plan, 33, 654-665. https://doi.org/10.1093/heapol/czy004

[26] Arnesen, L., Durán, P., Silva, J. and Brumana, L. (2016) A Multi-Country, Cross-Sectional Observational Study of Retinopathy of Prematurity in Latin America and the Caribbean TT-Estudio de observación, multinacional y transversal de la retinopatía del prematuro en América Latina y el Caribe. Revista Panamericana de Salud Pública, 39, 322-329.

[27] Quinn, G. (2016) Retinopathy of Prematurity Blindness Worldwide: Phenotypes in the Third Epidemic. Eye and Brain, 8, 31-36. https://doi.org/10.2147/EB.S94436

[28] Fortes Filho, J.B., Borges Fortes, B.G., Tartarella, M.B. and Procianoy, R.S. (2013) Incidence and Main Risk Factors for Severe Retinopathy of Prematurity in İnfants Weighing Less than 1000 Grams in Brazil. Journal of Tropical Pediatrics, 59, 502-506. https://doi.org/10.1093/tropej/fmt036

[29] Shah, P.K., Narendran, V., Kalpana, N. and Gilbert, C. (2009) Severe Retinopathy of Prematurity in Big Babies in India: History Repeating İtself? Indian Journal of Pediatrics, 76, 801-804. https://doi.org/10.1007/s12098-009-0175-1

[30] Ugurbas, S.C., Gulcan, H., Canan, H., Ankarali, H., Torer, B. and Akova, Y.A. (2010) Comparison of UK and US Screening Criteria for Detection of Retinopathy of Prematurity in a Developing Nation. Journal of AAPOS, 14, 506-510. https://doi.org/10.1016/j.jaapos.2010.07.012

[31] Akman, I., Demirel, U., Yenice, O., Ilerisoy, H., Kazokoğlu, H. and Ozek, E. (2010) 
Screening Criteria for Retinopathy of Prematurity in Developing Countries. European Journal of Ophthalmology, 20, 931-937. https://doi.org/10.1177/112067211002000519

[32] Akçakaya, A.A., Yaylali, S.A., Erbil, H.H., Sadigov, F., Aybar, A., Aydin, N., et al. (2012) Screening for Retinopathy of Prematurity in a Tertiary Hospital in Istanbul: Incidence and Risk Factors. Journal of Pediatric Ophthalmology and Strabismus, 49, 21-25. https://doi.org/10.3928/01913913-20110208-01

[33] Sahin, A., Sahin, M., Türkcü, F.M., Cingü, A.K., Yüksel, H., Cinar, Y., et al. (2014) Incidence of Retinopathy of Prematurity in Extremely Premature İnfants. ISRN Pediatrics, 2014, Article ID: 134347.

[34] Başmak, H., Niyaz, L., Şahin, A., Erol, N. and Gürsoy, H.H. (2010) Retinopathy of Prematurity: Screening Guidelines Need to Be Reevaluated for Developing Countries. European Journal of Ophthalmology, 20, 752-755.

https://doi.org/10.1177/112067211002000417

[35] Holmström, G., Hellström, A., Jakobsson, P., Lundgren, P., Tornqvist, K. and Wallin, A. (2015) Evaluation of New Guidelines for ROP Screening in Sweden Using SWEDROP-A National Quality Register. European Journal of Ophthalmology, 93, 265-268. https://doi.org/10.1111/aos.12506

[36] Tabarez-Carvajal, A.C., Montes-Cantillo, M., Unkrich, K.H., Trivedi, R.H. and Peterseim, M.M.W. (2017) Retinopathy of Prematurity: Screening and Treatment in Costa Rica. British Journal of Ophthalmology, 101, 1709-1713. https://doi.org/10.1136/bjophthalmol-2016-310005

[37] Chaudhry, T.A., Hashmi, F.K., Salat, M.S., Khan, Q.A., Ahad, A., Taqui, A.M., et al. (2014) Retinopathy of Prematurity: An Evaluation of Existing Screening Criteria in Pakistan. British Journal of Ophthalmology, 98, 298-301. https://doi.org/10.1136/bjophthalmol-2013-304018

[38] Bas, A.Y., Koc, E., Dilmen, U., Oguz, S.S., Ovali, F., Demirel, N., et al. (2015) Incidence and Severity of Retinopathy of Prematurity in Turkey. British Journal of Ophthalmology, 99, 1311-1314. https://doi.org/10.1136/bjophthalmol-2014-306286

[39] Early Treatment for Retinopathy of Prematurity Cooperative Group (2003) Revised Indications for the Treatment of Retinopathy of Prematurity. Archives of Ophthalmology, 121, 1684-1694. https://doi.org/10.1001/archopht.121.12.1684

[40] Gezer, A., Sezen, F., Şerifoğlu, I. and Karaçorlu, M. (1999) Management of Retinopathy of Prematurity with Cryotherapy. European Journal of Ophthalmology, 9 , 49-52.

[41] Mehmet, S., Fusun, A., Sebnem, C., Ozgur, O., Gulten, E., Taylan, O.A., et al. (2011) One-Year Experience in the Retinopathy of Prematurity: Frequency and Risk Factors, Short-Term Results and Follow-Up. International Journal of Ophthalmology, 4, 634-640. 